\title{
Prediction of Prolonged Hemodynamic Instability During Carotid Angioplasty and Stenting
}

\author{
Jong Kook Rhim, MD', Jin Pyeong Jeon, MD², Jeong Jin Park, MD³, Hyuk Jai Choi, MD², \\ Young Dae Cho, MD', Seung Hun Sheen, MD', Kyung-Sool Jang, MD ${ }^{6}$
}

Purpose: The aim of this study was to assess the risk factors of prolonged hemodynamic instability (HDI) after carotid angioplasty and stenting (CAS). Herein, a simplified predictive scoring system for prolonged HDI is proposed.

Materials and Methods: Sixty-six patients who had CAS from 2011 to 2016 at a single institution were evaluated. Prolonged HDI was defined as systolic blood pressure $>160 \mathrm{~mm} \mathrm{Hg}$ or $<90 \mathrm{~mm} \mathrm{Hg}$ or heart rate $<50$ beats/min, lasting over 30 minutes despite medical treatments. For the study, clinical data and radiologic data, including plaque morphology and stenosis were analyzed.

Results: Prolonged HDI was observed in 21 patients (31.8\%). Multivariable analysis revealed that calcification (OR, 6.726; $p=0.006)$, eccentric stenosis ( $O R, 3.645 ; p=0.047)$ and extensive plaque distribution (OR, 7.169; $p=0.006)$ were related to prolonged HDI. According to these results, a simplified scoring scale was proposed based on the summation of points: 2 points for calcified plaque, 2 points for extensive plaque distribution, and 1 point for eccentric stenosis. The percentages of prolonged $\mathrm{HDI}$ according to the total score were as follows: score $0,8.7 \%$; score $1,20.0 \%$; score 2 , $38.5 \%$; score 3, $72.7 \%$; score 4, $66.7 \%$; score $5,100 \%$. From the analysis, the total score in patients with prolonged HDI was significantly higher than those without prolonged HDI $(p<0.001)$.

Conclusion: Prolonged HDI can be associated with calcification of plaque, eccentric stenosis and extensive plaque distribution, and a simplified scoring system enables prediction of prolonged HDI according to our cohort.

Key Words : Carotid angioplasty and stenting; Hemodynamic instability; Stent

\footnotetext{
'Department of Neurosurgery, Jeju National University College of Medicine, Jeju, Korea

2Department of Neurosurgery, Hallym University College of Medicine, Chuncheon, Korea

${ }^{3}$ Department of Neurology, Konkuk University College of Medicine, Seoul, Korea

${ }^{4}$ Department of Radiology, Seoul National University College of Medicine, Seoul, Korea

${ }^{5}$ Department of Neurosurgery, Bundang Jesaeng Hospital, Bundang, Korea

${ }^{6}$ Department of Neurosurgery, Catholic University College of Medicine, Incheon, Korea

Received August 24, 2016; accepted after revision August 25, 2016.
}

Correspondence to: Jin Pyeong Jeon, MD, Department of Neurosurgery, Hallym University College of Medicine, 77 Sakju-ro, Chuncheon 200704 , Korea.

Tel. 82.33.240.5171Fax.82.33.240.9970 E-mail: jjs6553@daum.net

This is an Open Access article distributed under the terms of the Creative Commons Attribution Non-Commercial License (http://creativecommons.org/licenses/by-nc/3.0) which permits unrestricted non-commercial use, distribution, and reproduction in any medium, provided the original work is properly cited. 
Carotid angioplasty and stenting (CAS) has been widely performed due to its less invasive nature and simplicity compared to carotid endarterectomy (CEA) [1-3]. During CAS, however, hemodynamic instability (HDI) of hypertension, hypotension or bradycardia can happen due to manipulation near the carotid sinus and adventitial baroreceptors.

The frequency of HDI has been reported to occur in up to $42.4 \%$ of cases [4]. Qureshi et al. [5] also categorized the post-procedural rates of HDI: $22.4 \%$ hypotension, $27.5 \%$ bradycardia and $38.8 \%$ hypertension. Risk factors of HDI have been well described; however, most results have not considered the duration of HDI. Recovery from transient changes in HDI can be adequately done by immediate cardiac pacing or medical treatments. Accordingly, more emphasis should be placed on resolving prolonged HDI due to a higher probability of neurologic complications. The goal of this study was to investigate the risk factors of prolonged HDI, focusing on plaque and stenosis characteristics. In addition, we introduced a predictive scoring system for prolonged HDI after CAS.

\section{MATERIALS AND METHODS}

\section{Patient Sample}

This retrospective analysis was performed in patients who underwent CAS from 2011 to August 2016 at a single institution. A total of 72 patients underwent CAS during this period. After excluding 5 cases, which were done under emergent situations, and one case, which was lost to follow-up, 66 patients were included in this study. Clinical data such as sex, age, hypertension (HTN), diabetes mellitus (DM), coronary artery disease (CAD), coagulopathy, and the presence of symptoms were reviewed. Radiologic data were reviewed concerning calcification, distribution, ulceration, stenosis degree, and contralateral occlusion. Carotid plaque calcification was referred to as a structure with a density greater than 130 Hounsfield unit within the vessel wall that was hyperdense to the contrast-enhanced lumen and surrounding parenchyma on axial carotid CT [6]. Plaque distributions were assessed on the lateral projection image of digital subtraction angiography. Plaque located from CCA (common carotid artery) to ICA (internal carotid artery) within $5 \mathrm{~mm}$ in length from both sides of bifurcation was defined as being an extensive plaque [7]. Maximal stenosis was measured by the NASCET [8] method. Stenotic types were divided into two groups, eccentric and concentric, based on symmetry on axial images of CTA or Dyna-CT, according to previous reports (eccentric vs. concentric) $[4,7,9]$. Prolonged HDI was defined as systolic blood pressure $>160 \mathrm{~mm} \mathrm{Hg}$ [5] or $<90 \mathrm{~mm} \mathrm{Hg}$ or heart rate $<50$ beats/min [10] lasting over 30 minutes, [7] despite adequate treatments such as administration of fluid or a vasopressor. Patients who did not have a hemodynamic change or had transient HDI were regarded as patients without prolonged HDI. Bradycardia was treated immediately by a transcutaneous temporary cardiac pacemaker [11]. Atropine (0.25 mg) was infused intravenously and was repeated if necessary. Hypotension was treated by fluid replacement and dopamine with a starting dose of $5 \mu \mathrm{g} / \mathrm{kg} / \mathrm{min}$. Intravenous labetalol, hydralazine or nicardipine was infused for hypertension. Radiologic data were recorded by two qualified endovascular neurosurgeons and discrepancies were reevaluated by a third reader. This study was approved by the institutional review board (IRB) of the medical center (No 2015-30).

\section{Protocol of CAS}

CAS was performed according to previously reported protocols [7]. Before the procedure, every patient had taken oral aspirin $(100 \mathrm{mg})$ and clopidogrel $(75 \mathrm{mg})$ for more than 7 days. All procedures were conducted under local anesthesia through a trans-femoral approach with an elective schedule. Each patient had a percutaneous cardiac pacing patch, which was set at $50 \mathrm{bpm} / 20 \mathrm{~mA}$ in order to control bradycardia [11]. The electrocardiogram, BP, and blood oxygen saturation were continuously monitored. After a 6-F guiding sheath was introduced, 3000 units of heparin were administered through an intravenous route. During the procedure, the heparin-mixed fluid was injected (1000 units of heparin in 1-L sodium chloride solution) continuously in order to flush the catheter. After a roadmap was established, a cerebral protection device [Spider FX (ev3 Endovascular, Inc., Plymouth, MN, USA) or FilterWire EZ (Boston Scientific Corporation, Natick, MA, USA)] was placed, distal to the stenotic lesion. An Amiia or Aviator balloon catheter (Cordis Corporation, Miami, FL, USA) was used for pre-stent dilatation at nominal atmosphere (ATM) in cases for which dilatation was needed. A PRECISE-RX (Cordis Corporation) or PROTÉGÉ (ev3 Endovascular, Inc.) stent was applied at the stenotic segment. Post-stent balloon dilatations were used, if necessary. Every angiogram was conducted using the Artis zee Biplane system (SIEMENS Corp., Berlin, Germany). After the procedure, patients were closely monitored at the 


\section{Jong Kook Rhim, et al.}

intensive care unit for 24 hours.

\section{Statistical Analysis}

Continuous data are presented as the mean \pm standard deviation (SD). Chi-square or Fisher's exact test, and independent t-test were used for categorical and continuous data, respectively. Univariate analysis for relevant factors of prolonged HDI was conducted.
Then the risk factors for prolonged HDI were determined with a binary logistic regression model with $\mathrm{p}<0.1$. A new simplified scoring system was derived from variables showing significant association with prolonged HDI in the multivariable analyses. A Mann-Whitney U-test was conducted to compare the unpaired continuous data. P-values less than 0.05 were regarded as statistically significant. Statistics were

Table 1. Risk Factor Analysis of Prolonged Hemodynamic Instability (HDI) During Carotid Angioplasty and Stenting ( $\mathrm{n}=66)$

\begin{tabular}{|c|c|c|c|}
\hline \multirow{2}{*}{ Variables } & \multirow{2}{*}{ Without prolonged HDI $(n=45)$} & \multirow{2}{*}{ Prolonged HDI $(n=21)$} & \multirow{2}{*}{$\begin{array}{c}\text { Univariable analysis } \\
\text { p-value }\end{array}$} \\
\hline & & & \\
\hline Male & $39(86.7 \%)$ & $17(81.0 \%)$ & 0.546 \\
\hline Age, years & $67.9 \pm 11.0$ & $72.1 \pm 6.3$ & 0.103 \\
\hline HTN & 29 (64.4\%) & $16(76.2 \%)$ & 0.340 \\
\hline DM & $16(35.6 \%)$ & $10(47.6 \%)$ & 0.350 \\
\hline CAD & $6(13.3 \%)$ & $2(9.5 \%)$ & 0.659 \\
\hline Hyperlipidemia & $6(13.3 \%)$ & $1(4.8 \%)$ & 0.416 \\
\hline Contralateral occlusion & $8(17.8 \%)$ & $4(19.0 \%)$ & 0.901 \\
\hline Side of lesion (Lt.) & $25(55.6 \%)$ & $9(42.9 \%)$ & 0.336 \\
\hline Maximal stenosis (\%) & $81.4 \pm 9.3$ & $79.8 \pm 12.9$ & 0.565 \\
\hline Symptomaticity & $38(84.4 \%)$ & $19(90.5 \%)$ & 0.506 \\
\hline Calcification & $8(17.8 \%)$ & $10(47.6 \%)$ & 0.011 \\
\hline Ulceration & $9(20.0 \%)$ & $5(23.8 \%)$ & 0.724 \\
\hline Eccentric stenosis & $15(33.3 \%)$ & $12(57.1 \%)$ & 0.067 \\
\hline Extensive plaque & $5(11.1 \%)$ & 9 (42.9\%) & 0.003 \\
\hline Balloon time (sec) & $21.4 \pm 8.8$ & $14.2 \pm 8.0$ & 0.266 \\
\hline Balloon size (mm) & $4.3 \pm 0.5$ & $4.2 \pm 0.4$ & 0.239 \\
\hline Balloon length $(\mathrm{mm})$ & $28.9 \pm 6.5$ & $28.1 \pm 4.0$ & 0.608 \\
\hline Stent size $(\mathrm{mm})$ & $7.3 \pm 1.2$ & $7.0 \pm 1.3$ & 0.268 \\
\hline Stent length (mm) & $46.9 \pm 10.0$ & $43.8 \pm 8.0$ & 0.220 \\
\hline Maximal ATM & $9.5 \pm 3.4$ & $10.0 \pm 2.3$ & 0.513 \\
\hline
\end{tabular}

ATM, Atmosphere; HTN, Hypertension

$\mathrm{OR}$, odds ratio; $\mathrm{Cl}$, confidence interval

${ }^{*} \mathrm{P}<.05$ is significant.

Table 2. Multivariate Analysis for Risk Factors of Prolonged Hemodynamic Instability

\begin{tabular}{lccc}
\hline Variables & Odds ratio & $95 \% \mathrm{Cl}$ & $\mathrm{p}$-value \\
\hline Calcification & 6.726 & $1.725-26.219$ & $0.006^{*}$ \\
Eccentric stenosis & 3.645 & $1.017-13.072$ & $0.047^{*}$ \\
Extensive plaque & 7.169 & $1.741-29.520$ & $0.006^{*}$ \\
\hline
\end{tabular}

OR, odds ratio; $\mathrm{Cl}$, confidence interval

${ }^{*} \mathrm{P}<.05$ is significant. 
performed with SPSS (version 18; IBM Corporation, Chicago, IL, USA).

\section{RESULTS}

A total 66 patients were included in this analysis. The mean age of the patients was $69.2 \pm 9.9$ years and 56 $(84.8 \%)$ of the patients were male. Thirty-four patients $(51.5 \%)$ had stenosis on the left side. Symptomatic lesion was observed in 57 patients $(86.4 \%)$. Regarding plaque characteristics, 18 cases $(27.3 \%)$ showed calcification and 14 cases $(21.2 \%)$ had ulceration. Contralateral occlusion was noted in 12 patients $(18.2 \%)$. In 14 cases $(21.2 \%)$, extensive plaque was observed.

\section{Risk factors of prolonged HDI}

Prolonged HDI was observed in 21 patients $(31.8 \%)$. Hypotension occurred in 11 patients $(16.7 \%)$,

Table 3. A New Predictive Scoring System for Prolonged Hemodynamic Instability after Carotid Angioplasty and Stenting

\begin{tabular}{lcl}
\hline Variables & Points & Description \\
\hline Plaque morphology & 2 & Calcification \\
\hline Plaque distribution & 2 & Extensive location \\
\hline Type of stenosis & 0 & Proximal or distal location \\
\hline Total score & 1 & Eccentric features \\
\hline
\end{tabular}

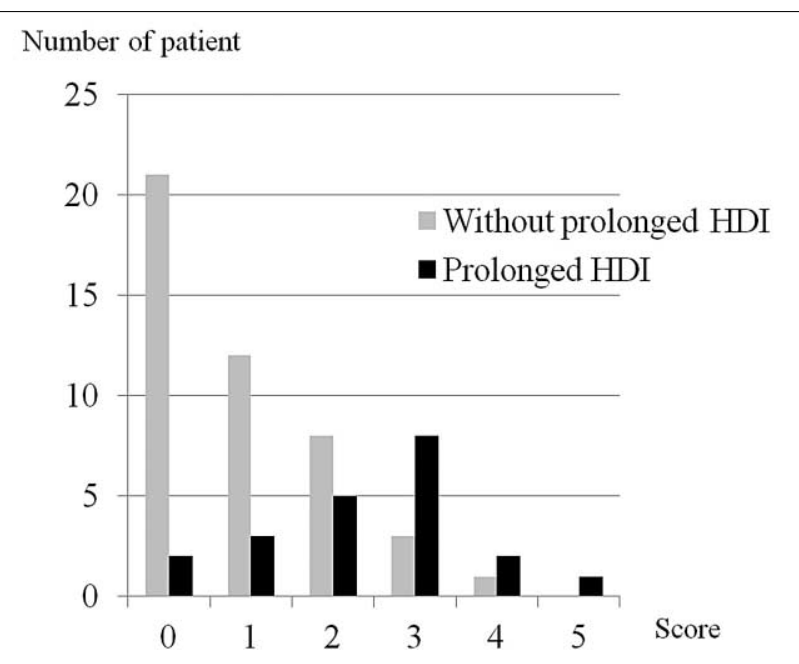

Fig. 1. Probability of prolonged hemodynamic instability in our cohort stratified by risk score. bradycardia in 7 patients $(10.6 \%)$, and hypertension in 5 patients $(7.6 \%)$. For patients with prolonged HDI, two cases $(9.5 \%)$ of symptomatic ischemic insults and one case $(4.8 \%)$ of seizure were observed. The remaining patients without prolonged HDI, two patients (4.4\%), complained of transient ischemic symptoms, such as verbal fluency and motor weakness. There was no hemorrhagic event in our series. A univariate analysis showed that calcification $(\mathrm{p}=0.011)$ and extensive plaque distribution $(\mathrm{p}=0.003$ ) were associated with prolonged HDI. Eccentric stenosis was more noted in prolonged HDI, but was not statistically significant $(\mathrm{p}=0.067)$. Other variables such as ulceration $(p=0.724)$, symptomatic lesion $(p=0.506)$, lesion side $(p=0.336)$ and contralateral occlusion $(p=0.901)$ did not have statistical significance (Table 1). Multivariate analysis revealed that calcification (OR, 6.726; $\mathrm{p}=0.006)$, eccentric stenosis $(\mathrm{OR}, 3.645 ; \mathrm{p}=0.047)$ and extensive plaque distribution (OR, $7.169 ; \mathrm{p}=0.006)$ were related to prolonged HDI (Table 2).

\section{A simplified scoring system for prolonged HDI}

A new simplified predictive scoring system was developed to estimate prolonged HDI in patients with multiple risk factors. After obtaining the relevant factors of calcification, eccentric stenosis, and extensive plaque distribution by multivariate analysis, a simplified risk score was established. Calcified plaque was defined as 2 points, and without calcification as 0 points. Extensive plaque distribution was defined as 2

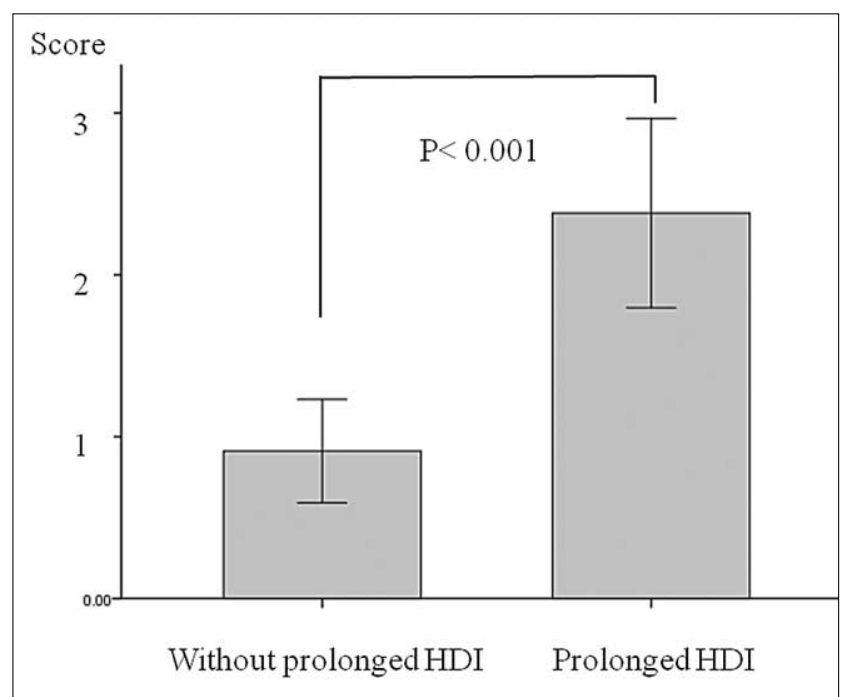

Fig. 2. Graph demonstrating statistical difference in risk scores between two groups, with or without prolonged hemodynamic instability, after carotid angioplasty and stenting. The total score in patients with prolonged $\mathrm{HDI}(2.4 \pm 1.3)$ is significantly higher than those without prolonged HDI $(0.9 \pm 1.1)(p<0.001)$. 


\section{Jong Kook Rhim, et al.}

points and a proximal or distal plaque as 0 points. Eccentric stenosis was defined as 1 point, and concentric stenosis as 0 points (Table 3 ). The proportion of prolonged HDI development according to the total scores was as follows: $8.7 \%$ for a score of 0 in 23 patients; $20.0 \%$ for a score of 1 in 15 patients; $38.5 \%$ for s score of 2 in 13 patients; $72.7 \%$ for a score of 3 in 11 patients; $66.7 \%$ for a score of 4 in 3 patients; and $100 \%$ for a score of 5 in one patient (Fig. 1). The total score in patients with prolonged HDI $(2.4 \pm 1.3)$ was significantly higher than those without prolonged HDI $(0.9 \pm 1.1)(\mathrm{p}<0.001)$ (Fig. 2).

\section{DISCUSSION}

HDI can develop though activation of adventitial baroreceptors located in the carotid sinus [12, 13]. Baroreceptors are stretch receptors providing shortterm regulation of abrupt hemodynamic change. The receptors activity is regulated by arterial pressure. When deploying a stent or an inflating balloon, impulse from the activated baroreceptors lead to the activation of nerve fibers to the nucleus tracts solitaries through the glossopharyngeal nerve and stimulate parasympathetic activation. Parasympathetic regulation of the cardiac nodes results in a lowering of heartbeat and peripheral resistance [5]. Although baroreceptors have a short-lasting effect, prolonged HDI can occur due to prolonged sinus activation and predominant sympathetic activity after the procedures. Prolonged HDI can result in serious complications including acute cerebral infarction, intracranial hemorrhage (ICH), myocardial infarctions, and renal failure $[14,15]$. Accordingly, hemodynamic stabilization is important during and after interventional procedures, especially for patients at a high risk for HDI.

HDI has been reported to be associated with female gender [5], old age [16], symptomatic lesion, stenotic lesion near the carotid bulb, posterior carotid plaque, ulcerated atherosclerotic plaque, calcified plaque, contralateral occlusion, a previous history of CEA and general anesthesia [17-21]. However, most results from previous studies have focused on risk factors of HDI development itself, without considering HDI-holding time. Transient hemodynamic changes can be treated well by medical treatments without complications. Accordingly, studies on preventing prolonged HDI could be more important than those on transient HDI.

An eccentric type of stenosis and calcification at carotid bifurcation has been reported to be associated with prolonged hypotension that lasts over 3 hours [4].
Baroreceptor activation is affected by the rate of pressure change as well as its degree. Calcification and eccentric location of plaque may lead to uneven pressure which is transmitted to arterial walls through increased inflation rate, in particular to arterial walls where the plaque is not located $[4,7]$. Our result also showed that prolonged HDI was related to the existence of eccentric stenosis (OR, 3.645; $\mathrm{p}=0.047)$ and calcified plaque $(\mathrm{OR}, 6.726 ; \mathrm{p}=0.006)$. In this study, we defined extensive plaque as atherosclerotic carotid plaque, which was located in CCA to ICA within $5 \mathrm{~mm}$ in length from both sides of bifurcation [7]. Although baroreceptors are often observed in the proximal ICA just above the carotid bifurcation, baroreceptors are also found in the distal CCA, near the carotid bifurcation $[7,22,23]$. Nevertheless, the presence of inclusion of stenotic lesions in the carotid bulb has been the focus. Our result showed that extensive plaque located from CCA to carotid bulb was associated with prolonged HDI (OR: 7.169; $\mathrm{p}=0.006)$.

ICH develops ranging from $0.63 \%$ to $2.69 \%$ in incidence after CAS and is related to significant mortality and morbidity [24, 25] Many researchers have thought that cerebral hyperperfusion syndrome (HFS) can be related to ICH after the procedures [24, 25]. Post-procedural hypertension and decreased cerebrovascular reserve capacity can result in HFS [26]. Qureshi et al. [5] reported that the rate of hypertension over $160 \mathrm{mmHg}$ was noted in $38.8 \%$ of cases after CAS. We also experienced a 7.6\% (5 out of 66) rate of hypertension. Xu et al. [24] reported that the mean time of ICH diagnosis was $6.1 \pm 7.1$ hours after the procedures. Accordingly, strict blood pressure control until improved cerebral autoregulation is necessary in the peri-operative period. Nevertheless, the optimal time for strict blood pressure control remains unclear due to differences in impaired vascular reserve capacity among patients. In addition, ischemic changes due to decreased cerebral blood flow lead to severe brain damage. Therefore, further imaging studies to find the reserve capacity and autoregulation in maintaining blood pressure control are required [24].

Stent cell design can be related to procedural outcomes due to different radial forces and properties of plaque stabilization. Bosiers et al. [27] reported that post-procedural complication rates are observed frequently in patients who undergo open cell types and with larger free cell area. The authors thought that small free cell area dense meshes are beneficial to stabilization after CAS [27, 28]. The open-cell stents yielded high flexibility and adaptability to the arterial 
wall, which allowed particle penetration through the open struts [29]. We used PRECISE-RX or PROTÉGÉ stents. Although risk of neurologic complications, such as stroke and mortality, did not differ significantly according to stent types (closed versus open-cell design) [28], risk factor analyses of prolonged HDI with regard to the stent design are further needed.

Many patients have multiple risk factors for HDI during clinical procedures. In our cohort, 15 patients (22.7\%) had multiple risk factors of prolonged HDI. In such cases, counseling patients with future development of hemodynamic change can be difficult. Therefore, we created a new simplified scoring scale for predicting the prolonged HDI. The proportion of prolonged HDI according to risk score was as follows: $8.7 \%$ for a score of 0 in 23 patients; $20.0 \%$ for a score of 1 in 15 patients; $38.5 \%$ for a score of 2 in 13 patients; $72.7 \%$ for a score of 3 in 11 patients; $66.7 \%$ for a score of 4 in 3 patients; and 100\% for a score of 5 in one patient. Accordingly, patients at a high risk for prolonged HDI need a treatment strategy, such as avoidance of sudden activation of baroreceptors [7]. In such cases, suboptimal expansion without post-stent balloon expansion [30] and preventive therapies to increase circulating volume can be beneficial [7].

For this study, concerns remain about the possibility of selection bias due to its retrospective nature and having been conducted at a single institution. Also, the scoring system that was proposed was base on a singlecenter experience with 66 subjects. Accordingly, external validation is necessary in prospective multicenter studies to confirm our results.

In conclusion, prolonged HDI can be associated with calcification of plaque, eccentric stenosis and extensive plaque distribution. A simplified scoring system enables prediction of prolonged HDI in our cohort. External validation through prospective multi-center observational studies is required to confirm our results.

\section{Acknowledgement}

This study was supported by a grant from Korean Society of Interventional Neuroradiology. We would like to thank Sung-Eun Kim for her help with the data collection and statistical support.

\section{References}

1. Dietz A, Berkefeld J, Theron JG, Schmitz-Rixen T, Zanella FE, Turowski B, et al. Endovascular treatment of symptomatic carotid stenosis using stent placement: long-term follow-up of patients with a balanced surgical risk/benefit ratio. Stroke 2001;32:18551859
2. Brown MM, Venables G, Clifton A, Gaines P, Taylor RS. Carotid endarterectomy vs carotid angioplasty. Lancet 1997;349:880-881

3. Brooks WH, McClure RR, Jones MR, Coleman TL, Breathitt L. Carotid angioplasty and stenting versus carotid endarterectomy for treatment of asymptomatic carotid stenosis: a randomized trial in a community hospital. Neurosurgery 2004;54:318-324

4. Nonaka T, Oka S, Miyata K, Mikami T, Koyanagi I, Houkin K, et al. Prediction of prolonged postprocedural hypotension after carotid artery stenting. Neurosurgery 2005;57:472-477

5. Qureshi AI, Luft AR, Sharma M, Janardhan V, Lopes DK, Khan $\mathrm{J}$, et al. Frequency and determinants of postprocedural hemodynamic instability after carotid angioplasty and stenting. Stroke 1999;30:2086-2093

6. Nandalur KR, Baskurt E, Hagspiel KD, Finch M, Phillips CD, Bollampally SR, et al. Carotid artery calcification on CT may independently predict stroke risk. AJR Am J Roentgenol 2006;186:547-552

7. Jeon JS, Sheen SH, Hwang G. Hemodynamic instability during carotid angioplasty and stenting-relationship of calcified plaque and its characteristics. Yonsei Med J 2013;54:295-300

8. North American Symptomatic Carotid Endarterectomy Trial Collaborators. Beneficial effect of carotid endarterectomy in symptomatic patients with high-grade carotid stenosis. $N$ Engl J Med 1991;325:445-453

9. Waller BF. The eccentric coronary atherosclerotic plaque: morphologic observations and clinical relevance. Clin Cardiol 1989;12:14-20

10. Cieri E, De Rango P, Maccaroni MR, Spaccatini A, Caso V, Cao $\mathrm{P}$. Is haemodynamic depression during carotid stenting a predictor of peri-procedural complications? Eur J Vasc Endovasc Surg 2008;35:399-404

11. Im SH, Han MH, Kim SH, Kwon BJ. Transcutaneous temporary cardiac pacing in carotid stenting: noninvasive prevention of angioplasty-induced bradycardia and hypotension. J Endovasc Ther 2008;15:110-116

12. Chalmers J, Pilowsky P. Brainstem and bulbospinal neurotransmitter systems in the control of blood pressure. J Hypertens 1991;9:675-694

13. Mangin L, Medigue C, Merle JC, Macquin-Mavier I, Duvaldestin $\mathrm{P}$, Monti A, et al. Cardiac autonomic control during balloon carotid angioplasty and stenting. Can J Physiol Pharmacol 2003;81:944-951

14. Leisch F, Kerschner K, Hofman R, Bibl D, Engleder C, Bergmann H. Carotid stenting: acute results and complications. Z Kardiol 1999;88:661-668

15. Morrish W, Grahovac S, Douen A, Cheung G, Hu W, Farb R, et al. Intracranial hemorrhage after stenting and angioplasty of extracranial carotid stenosis. AJNR Am J Neuroradiol 2000;21: 1911-1916

16. Mlekusch W, Schillinger M, Sabeti S, Nachtmann T, Lang W, Ahmadi R, et al. Hypotension and bradycardia after elective carotid stenting: frequency and risk factors. $J$ Endovasc Ther 2003; 10:851-859

17. Wong JH, Findlay JM, Suarez-Almazor ME. Hemodynamic instability after carotid endarterectomy: risk factors and associations with operative complications. Neurosurgery 1997;41:35-41

18. Schulz UG, Rothwell PM. Sex differences in carotid bifurcation anatomy and the distribution of atherosclerotic plaque. Stroke 2001;32:1525-1531 


\section{Jong Kook Rhim, et al.}

19. Cayne NS, Faries PL, Trocciola SM, Saltzberg SS, Dayal RD, Clair D, et al. Carotid angioplasty and stent-induced bradycardia and hypotension: impact of prophylactic atropine administration and prior carotid endarterectomy. J Vasc Surg 2005;41:956-961

20. Dangas G, Laird JR, Jr., Satler LF, Mehran R, Mintz GS, Larrain $\mathrm{G}$, et al. Postprocedural hypotension after carotid artery stent placement: predictors and short- and long-term clinical outcomes. Radiology 2000;215:677-683

21. Taha MM, Toma N, Sakaida H, Hori K, Maeda M, Asakura F, et al. Periprocedural hemodynamic instability with carotid angioplasty and stenting. Surg Neurol 2008;70:279-285

22. Fadel PJ. Arterial baroreflex control of the peripheral vasculature in humans: rest and exercise. Med Sci Sports Exerc 2008;40:20552062

23. Fadel PJ, Ogoh S, Keller DM, Raven PB. Recent insights into carotid baroreflex function in humans using the variable pressure neck chamber. Exp Physiol 2003;88:671-680

24. Xu Y, Wanga Y, Feng L, Miao Z, Ling F. Treatment and outcome of intracranial hemorrhage after carotid artery stenting. A ten year single center experience. Interv Neuroradiol 2009;15: 316-324

25. Kang HS, Han MH, Kwon OK, Kwon BJ, Kim SH, Oh CW.
Intracranial hemorrhage after carotid angioplasty: a pooled analysis. J Endovasc Ther 2007;14:77-85

26. van Mook WN, Rennenberg RJ, Schurink GW, van Oostenbrugge RJ, Mess WH, Hofman PA, et al. Cerebral hyperperfusion syndrome. The Lancet Neurology 2005;4:877-888

27. Bosiers M, de Donato G, Deloose K, Verbist J, Peeters P, Castriota F, et al. Does free cell area influence the outcome in carotid artery stenting? Eur J Vasc Endovasc Surg 2007;33:135141

28. Schillinger M, Gschwendtner M, Reimers B, Trenkler J, Stockx L, Mair J, et al. Does carotid stent cell design matter? Stroke 2008;39:905-909

29. Muller-Hülsbeck S, Schäfer PJ, Charalambous N, Schaffner SR, Heller M, Jahnke T. Comparison of carotid stents: an in-vitro experiment focusing on stent design. J Endovasc Ther 2009;16: 168-177

30. Jin SC, Kwon OK, Oh CW, Jung C, Han MG, Bae HJ, et al. A technical strategy for carotid artery stenting: suboptimal prestent balloon angioplasty without poststenting balloon dilatation. Neurosurgery 2010;67:1438-1442 